\title{
JUAN DUNS ESCOTO, QUAESTIONES SOBRE LA METAFÍSICA DE ARISTÓTELES LIBRO I
}

\author{
Traducción de Héctor Hernando Salinas \\ Pontificia Universidad Javeriana, Bogotá
}

\section{[I. SOBRE LA NOBLEZA DE LA CIENCIA METAFÍSICA]}

Todos los hombres desean saber por naturaleza...2

1. En el comienzo de la Metafísica, obra que tenemos entre las manos, el Filósofo presenta esta proposición que expresa, como será evidente en lo que sigue, la dignidad y la nobleza de esta ciencia. Para ver esto, en primer lugar hay que aclarar la proposición en sí y, luego, en segundo lugar, hay que aplicarla a la conclusión que buscamos.

\section{[A. Aclaración de la proposición]}

2. Esta proposición se aclara de dos maneras, a saber, a partir de lo posterior (a posteriori) y a partir de lo anterior (a priori).

\section{[1. A posteriori]}

3. En el texto, el Filósofo aclara la proposición, a partir de lo posterior, por un signo, cuando afirma: ${ }^{3}$ «En efecto, el signo es el amor a los sentidos; pues además de su utilidad, los sentidos se aman a causa de sí mismos» (Signum autem est sensuum dilectio; praeter enim utilitatem, propter se ipsos diliguntur); es como si dijera que los sentidos se aman naturalmente, no solo en cuanto son útiles para la sustentación de la vida, sino en cuanto son potencias cognitivas (sed in quantum cognoscitivi).

Lo prueba en el texto, ${ }^{4}$ en cuanto el sentido que se ama en grado sumo (maxime) es aquel que es en grado sumo cognitivo, a saber, la vista. Llama a este sentido «cognitivo en grado sumo» (maxime cognoscitivum) por dos razones: a causa de la certeza del conocimiento y de

1 Ioannis Duns Scoti, Questiones super Libros Metaphysicorum Aristotelis, Libro I-V, ed. R. Andrews et al., en: Opera philosophica III, St. Bonaventure (NY), The Franciscan Institut, 1997, p. 3-14. Esta traducción es uno de los resultados del proyecto de investigación 006268 Juan Duns Escoto antes de la univocidad del ser, inscrito en la Vicerrectoría de Investigación de la Pontificia Universidad Javeriana, Bogotá.

2 Aristóteles, Metaphysica, AL (Aristoteles Latinus) XXV² p. 7; I (A) c. 1, 980a 21.

3 Aristóteles, Metaphysica, AL XXV² p. 7; I (A) c. 1, 980a 21-23.

4 Aristóteles, Metaphysica, AL XXV² p. 7; I (A) c. 1, 980a 25-27. 
la multitud de objetos conocidos. La certeza proviene de su inmaterialidad: en efecto, en cuanto más inmaterial es una potencia cognitiva (virtus cognoscitiva), ofrece una mayor certeza en el acto de conocer. También se conocen muchos objetos por el sentido, porque todos los cuerpos tanto los superiores como los inferiores participan de la luz y el color; sin embargo, no de otras cualidades sensibles, a saber, las tangibles o audibles, y las otras.

4. La fuerza de la prueba de Aristóteles se encuentra aquí: si amamos naturalmente más, el sentido que es más cognitivo, y no solo a causa de su utilidad para la vida, sino a causa del conocimiento, de allí se sigue que naturalmente deseemos más saber (scire magis naturaliter desideramus), porque este conocimiento es más noble que el conocimiento sensible y nuestro conocimiento sensible se ordena a este como a su fin.

\section{[2. A priori: la opinión de Tomás de Aquino]}

5. En segundo lugar, algunos aclaran ${ }^{5}$ la proposición precitada por lo anterior o por la causa (a priori sive a causa), de tres maneras. En primer lugar, así: todo imperfecto naturalmente desea (appetit) su perfección, como puede verse en la Física I, último capítulo: ${ }^{6}$ en efecto, el alma del hombre es de suyo (de se) imperfecta según su potencia intelectiva, puesto que es como un tablero en blanco (tabula nuda) en el cual nada hay pintado, según el Filósofo en el libro Sobre el alma III. Por lo tanto, desea naturalmente saber, y esto es su perfección según esta potencia.

6. En segundo lugar se aclara así: Cada uno naturalmente desea (appetit) su propia operación, como lo pesado desea moverse hacia abajo. En efecto, la operación propia del hombre es entender (intelligere), puesto que por ella se distingue de los otros.

7. En tercer lugar, así: cada uno naturalmente desea (appetit) unirse a su principio. En efecto, el hombre se une a las substancias separadas por el acto de entender (per intelligere), como lo prueba el Filósofo en el libro X de la Ética, capítulo $10,{ }^{8}$ porque en la contemplación de la verdad (in speculatione veritatis) nos asimilamos en grado sumo (maxime) a las substancias separadas, y concluye que en esto consiste nuestra felicidad (beatitudinem); por lo tanto, el hombre naturalmente desea saber.

8. [Sobre la opinión de Tomás] Sin embargo, no parece que estas tres pruebas, en tanto sean válidas, se distingan mucho entre ellas:

Puesto que, como hay dos actos, el primero y el segundo, como es manifiesto a partir del libro II del tratado Sobre el alma, ${ }^{9}$ así también hay una doble perfección, la primera y la segunda. La primera es la forma o hábito, la segunda es la operación. Y cada uno naturalmente desea la perfección de la una y de la otra, ya que la operación es el fin del hábito. Por lo tanto, esta proposición «cada uno naturalmente desea su perfección» (§§ 5-6) implica la tesis a probar tanto para el caso de la ciencia, a propósito de la cual se ocupa la primera razón $<$ de Tomás>, como del entender (intelligere), a propósito del cual se ocupa la segunda razón

5 Tomás de Aquino, In Duodecim libros Metaphysicorum Aristotelis expositio, I, lectio I, nn. 2-4, ed. M. R. Cathala y R. M. Spiazzi, Turín-Roma, Marietti, 1950, p. 6.

6 Aristóteles, Physica, AL VII ${ }^{1}$ p. 39; I (A) c. 9, 192a 16-19.

7 Aristóteles, De anima, III (Г) c. 4, 429b 31-430a 2, en Tomás de Aquino, Sententia libri de anima, ed. R.-A. Gauthier, Opera Omnia, t. XLV.1,2 2a. Ed., Roma-París, Commissio Leonina-J. Vrin, 1984, p. 201.

8 Aristóteles, Ethica Nicomachea, $\mathrm{AL} \mathrm{XVI}^{3}$ p. 360; X (K) c. 7, 1177b 26-31.

9 Aristóteles, De anima, II (B) c. 1, 412a 10-11 (ed. Gauthier, p. 67). Cf. Auctoritates Aristotelis (ed. J. Hamesse, p. 177, n. 39): «Duplex est actus, scilicet primus et secundus: primus ut scientia, secundus ut speculari secundum scientiam». 
$<$ de Tomás $>$. Pues no parece que haya alguna necesidad segunda: ¿por qué razón se desea naturalmente la operación, si no es porque ella es una perfección?

9. Además, así como «potencia» se dice equívocamente de la potencia esencial que es a partir de la forma, y de la accidental que es a partir de la operación, como es manifiesto en el libro II del tratado Sobre el alma, ${ }^{10}$ así también parece que el término «deseo» (desiderium) sea equívoco a aquello que corresponde a la forma y a aquello que corresponde a la operación que sigue a la forma, pues en el primer caso el que desea no puede tener por sí lo deseado sin la acción de algún agente extrínseco; en el segundo caso sí puede, una vez removido todo impedimento. Por lo tanto, si la primera y la segunda prueba concluyen que esta proposición es verdadera, como pruebas distintas, se sigue que en esta proposición se toma «deseo» equívocamente.

10. Además, la tercera prueba no parece diferir de la primera, porque el hombre solo se une por naturaleza a su principio por su operación. Y no parece haber otra razón para desear la operación y tal unión.

11. A partir de estas tres pruebas se puede inferir una sola prueba: cada uno naturalmente desea su perfección, tanto la primera, que es la forma, como la segunda, que es su operación propia, por la cual se une a su principio. Por lo tanto, el hombre naturalmente desea la ciencia, que es su perfección primera, y entender, que es su perfección segunda, por la cual se une a su principio.

12. Y con el mismo deseo o, al menos, dicho unívocamente (como se expone más tarde), ${ }^{11}$ se desea (appetitur) la ciencia inmediatamente y entender (intelligere) mediante la ciencia. Y ciertamente este deseo corresponde a la potencia esencial, porque el que no tiene forma está en potencia esencial al acto primero y segundo.

13. Y también por el mismo deseo esencial puede desear inmediatamente la operación como su fin, y la forma a causa del fin, cuando no tiene ni el uno ni el otro, o, al menos, dicho unívocamente. En efecto, quizás no es la misma volición del ente al fin y el fin, en tanto el uno y el otro son aquello que se desea, sino solamente en tanto el fin es aquello por lo cual el otro se desea, como el color y la luz en la visión única no son vistos como objetos, sino el uno como «aquello en lo cual» (la luz) y el otro como «aquello que» (el color).

14. Sin embargo, en el otro deseo, y dicho equívocamente, se desea (appetitur) la ciencia inmediatamente y entender inmediatamente, cuando se habla del deseo accidental. El primero corresponde a la potencia esencial, el segundo a la accidental. El primero puede llamarse «deseo de la forma» (desiderium ad forman), el segundo «deseo que se sigue de la forma» (desiderium consequens formam).

15. Algunas objeciones contra esta proposición serán tratadas más adelante en algunas cuestiones. ${ }^{12}$ Pero esto es suficiente para aclararla.

\section{[16-21 B. Aplicación de la proposición al propósito]}

16. Ahora debemos aplicar la proposición a nuestro propósito, a saber, para demonstrar la dignidad y nobleza de esta ciencia, del siguiente modo: si todos los hombres desean por naturaleza saber, desearán por tanto en grado sumo la ciencia en grado sumo. Así argumenta el

10 Aristóteles, De anima, II (B) c. 5, 417a 26-28 (ed. Gauthier, p.110).

11 Duns Escoto, Questiones super Libros Metaphysicorum Aristotelis, vol. I, Libro I, q. 2, §§ 36-44, pp. 80-84.

12 Duns Escoto, Questiones super Libros Metaphysicorum Aristotelis, vol. I, Libro I, q. 2, §§ 30-42, pp. 79-83. 
Filósofo en el libro I capítulo $2 .^{13} \mathrm{Y}$ en el mismo lugar dice «que es ciencia en grado sumo (maxime sciencia)» aquella que se ocupa de las realidades cognoscibles en grado sumo (maxime scibilia). Las realidades cognoscibles en grado sumo se entienden de dos maneras: $<\mathrm{A}>$ porque son conocidas antes que todas las otras realidades (primo omnium) y sin ellas las otras realidades no pueden conocerse; $\langle\mathrm{B}>$ o porque son cognoscibles con máxima certeza (certissima cognoscibilia). Esta ciencia considera las realidades cognoscibles en grado sumo de los dos modos. Por lo tanto, esta es la ciencia en grado sumo y, en consecuencia, la ciencia deseable en grado sumo (maxime desiderabilis).

17. Prueba de las dos partes de la menor. Con respecto a la primera: las realidades cognoscibles en grado sumo (maxime scibilia) según el primer modo, son las realidades más comunes (communissima), como el ente en tanto ente, y todas aquellas realidades que se siguen (consequuntur) del ente en tanto ente. En efecto, Avicena afirma en su Metafisica I, capítulo $5 \mathrm{a}^{14}$ que «el ente y la cosa se imprimen en el alma por una impresión primera, que no se adquiere a partir de realidades más conocidas que ellas» (ens et res imprimuntur in anima prima impressione, quae non acquiritur ex aliis notioribus se). Y más abajo: «que las realidades anteriores que deben ser concebidas por sí mismas, son aquellas que son comunes a todas las cosas, como la cosa, el ente y el uno (sicut res et ens et unum). Y por lo tanto no puede demonstrarse por una prueba algo que pertence a ellos, que no sea $<$ por una prueba $>$ circular». Estas realidades comunísimas (haec communissima) competen a la consideración de la metafísica según el Filósofo al comienzo del libro IV: ${ }^{15}$ «hay una cierta ciencia que se ocupa del ente en tanto ente y de aquellas realidades que le pertenecen por sí» (Est scientia quaedam quae speculatur ens in quantum ens, et quae huic insunt secundum se), etc.

18. La necesidad de esta ciencia se prueba así: de la evidencia que las realidades comunísimas (communissima) se entienden (intelligunt) en primer lugar, como lo prueba Avicena, se sigue que las realidades especiales no pueden conocerse a menos que las realidades comunes se conozcan previamente. Y el conocimietno de estas realidades comunes no puede tratarse en una ciencia particular, porque por la misma razón podrían tratarse en una o en otra (puesto que el ente y el uno se dicen de modo equivalente de todas las cosas, como lo dice en el libro $\mathrm{X}),{ }^{16}$ de tal modo que se repetiría lo mismo inútilmente muchas veces. Por lo tanto, es necesario que haya una ciencia universal que considere por sí aquellas realidades trascendentes (quae per se consideret illa transcendentia). Y llamamos a esta ciencia «metafísica», cuyo nombre se construye a partir de «meta» que significa «trans»y de $« y \cos { }^{17}$ que significa

13 Aristóteles, Metaphysica, AL XXV² p. 10; I (A) c. 2, 982a 30-b 23.

14 Avicenna, Liber de philosophia prima sive Scientia divina I-IV, I c. 5, ed. S. Van Riet, intr. G. Verbeke, Lovaina-Lyden, Peeters-Brill, 1977, pp. 31-33.

15 Aristóteles, Metaphysica, AL XXV² p. 60; IV (Г) c. 1, 1003a 21-22.

16 Aristóteles, Metaphysica, AL XXV² p. 188; X (I) c. 1, 1053b 25-26.

17 La edición crítica (página 9, nota 20) presenta otros ejemplos del uso de esta etimología. Citemos aquí al autor anónimo del De cursu et libris studii, (Cod. Vaticanus latinus, 3022, f. 84va): «Yconomica dicitur ab yconomos quod est dispensare et ycos scientia, quasi scientia de dispensatione sui et suae familiae. Politica dicitur a polis quod est pluralitas et ycos scientia, quasi scientia de regimine plurium... Metaphysica dicitur a metha quod est trans et physis quod est natura, quasi transcendens naturam... Mathematica dicitur a matos auod est quantitas vel mensura et ycos scientia, quasi scientia de quantitate... et de tali est arismetica. Et dicitur ab aros quod est numerus et ycos scientia, quasi scientia de numero». En el comentario a su traducción, D. Demange («L'objet de la philosophie première selon Thomas d'Aquin et Jean Duns Scot», Le Philosophoire 20 (2003), p. 194) observa cómo Escoto se separa de la consideración de la metafísica como «transphysica», tal como lo hace Tomás en su propio prólogo. Escoto, por su parte, hace de la metafísica una ciencia trascendental en un sentido que no depende de la sola superación de la física ni se define por ella, sino por una universalidad mayor y por una superación de los objetos de todas las ciencias particulares gracias a la captación inmediata de los trascendentia según el preci- 
«ciencia», como una ciencia trascendental, porque es una ciencia que se ocupa de las realidades trascendentales. ${ }^{18}$ (Et hanc scientiam vocamus metaphysicam, quae dicitur a «meta», quod est «trans», et «ycos» «scientia», quasi transcendens scientia, quia est de transcendentibus).

19. [Adición posterior: $§ \S 19-20]$ ใ Esta prueba no parece eficaz, puesto que el ente se dice con anterioridad (per prius) de la substancia, según el libro IV. ${ }^{19}$ E igualmente el uno, si es un trascendental. Sin embargo, si solo es un género de la cantidad, como se trata de él en alguna cuestión del libro $\mathrm{IV},{ }^{20}$ de ese modo no se dice igualmente de todas las realidades (de omnibus). Para enteder esta proposición, ir al libro X, a los problemas. ${ }^{21}$

20. Respuesta: si el ente se dijera igualmente, la prueba sería evidente. También, si no se dijera igualmente, pero se dijera en primer lugar de algún ente (de uno aliquo), por ello en la ciencia de aquel primero se trataría de aquellos que son entes por sí, no en otra ciencia de algo posterior. Y así, como antes, habrá una única ciencia universal (una erit scientia universalis), no porque su sujeto sea universal en la predicación, sino según el orden de perfección. ใ१

21. La segunda parte de la menor (n. 16) se prueba así: las realidades cognoscibles con máxima certeza (certissima cognoscibilia) son principios y causas, y tanto más ciertas por sí cuanto más anteriores. De ellas depende toda certeza posterior. Esta ciencia considera de este modo los principios y las causas, como lo prueba el Filósoso en la Metafísica I, capítulo 2, ${ }^{22}$ en tanto llama a esta ciencia «sabiduría» (sapientia), como es manifiesto en el texto. Así, por tanto, es manifiesto cómo esta ciencia se ocupa de las realidades cognoscibles con máxima certeza (certissima cognoscibilia). De lo que se sigue que sea la ciencia en grado sumo y así la ciencia deseada en grado sumo, como ha sido deducido previamente (\$16).

\section{[II. SOBRE LAS CAUSAS DE ESTA CIENCIA]}

22. De lo dicho se pueden extraer tres causas de esta ciencia: final, formal y material.

No hay que preocuparse mucho de la causa eficiente instrumental, mientras la ciencia sea transmitida correctamente (sin embargo, se ha dicho que fue Aristóteles). Sin embargo, la causa eficiente principal es Dios, del cual se habla en el libro I, capítulo 3:23 «Solo Dios posee en grado sumo esta ciencia, aunque no la tenga él solo, pues envidiar no corresponde con su naturaleza», puesto que la envidia no se armoniza con la suma bondad. A causa de esta bondad, Dios quiso comunicar esta ciencia al hombre, así como otras perfecciones: «Toda verdad que es dicha por alguien, es dicha por el Espíritu Santo», como afirma la Glosa ${ }^{24}$ al primer

tado argumento de Avicena ( $\$ 17)$. En ese sentido, la metafísica como ciencia trascendental es de origen aviceniano, aunque en este prólogo no se consume plenamente su fundación. Ver la siguiente nota.

18 Sin embargo, no hay que olvidar que en el contexto de este prólogo, la metafísica como scientia transcendes es una de las dos dimensiones de la metafísica, pues en el parágrafo 21, Escoto introduce la metafísica como sapientia. Si la scientia transcendens se ocupa del ser y sus atributos, la sapientia se ocupa de los primeros principios y las primeras causas. En este prólogo, Escoto no va a tomar partido par una u otra dimensión como sujeto de la metafísica.

19 Aristóteles, Metaphysica, AL XXV² p. 60-61; IV (Г) c. 2, 1003b 6-10.

20 Duns Escoto, Questiones super Libros Metaphysicorum Aristotelis, vo. I, Libro IV, q. 2, §§ 93-126, p. 343-350.

21 Aristóteles, Metaphysica, AL XXV² p. 196-198; X (I) c. 6, 1056b 4-1057a 18

22 Aristóteles, Metaphysica, AL XXV² p. 9; I (A) c. 2, 982a 5-10.

23 Aristóteles, Metaphysica, AL XXV² p. 11; I (A) c. 2, 982b 29-983a 4.

24 Rábano Mauro, Expositio in epistolem I ad Corinthios, ed. Migne, Patrologia Latina CXII, p. 106C. 
libro de los Corintios (12,3): «Nadie puede decir señor Jesus», salvo el Espíritu Santo. Y Agustín, en las 83 cuestiones diversas, q. 1: «Todo lo verdadero es verdadero por la verdad primera». ${ }^{25}$ Y Juan Damasceno, en el capítulo 91: «Si de aquellos que están fuera», a saber, fuera de la Iglesia, «podemos hacer fructificar lo que es útil, no hay que rechazarlo, conservando el oro limpio y puro, y rechazando lo contaminado. Tomemos para nosotros los mejores discursos (sermones optimos); pero lancemos a los perros los ridículos dioses y las fábulas extranjeras. En efecto, de ellos y contra ellos, podremos poseer una gran fortaleza». ${ }^{26}$

23. Sobre la causa final hay que decir que el fin propio de esta ciencia es la operación según este hábito, a saber, la contemplación de las esencias de las cosas (speculatio essentiarum rerum) y principalmente de las altísimas causas y de las sustancias separadas, en la contemplación de las cuales, Aristóteles puso la felicidad, en el libro X de la Ética. ${ }^{27}$ Sin embargo, el fin extrínseco son la causa primera y las sustancias separadas, a las cuales se une nuestro intelecto mediante el hábito de esta ciencia. Sin embargo, esta ciencia no se ordena a otra como a su fin, sino que las otras se ordenan a ella. De allí que no es útil a otra ciencia, en cuanto el Filósofo en el texto ${ }^{28}$ dice «util», en el sentido de «ordenada a un fin», sino que es más digna y más noble como fin, que cualquier ciencia útil.

24. Sobre la causa formal hay que decir que la causa formal de esta ciencia es su modo de proceder (modus procedendi), que aquí (como en las otras ciencias) corresponde a tres procedimientos: dividir, definir y reunir.

25. [Adición posterior] १ใ En contra: dices más abajo (§ 26) que toda ciencia define y demuestra a través de rasgos esenciales (per essentialia). Respuesta: concedo esto; pero lo propio de esta ciencia será demostrar por las causas más universales y por las propiedades (passiones) más universales y por los rasgos esenciales de cualquier cosa, pero no en cuanto «esta», sino en cuanto substancial. १ใ.

26. Sin embargo, propio de esta ciencia es dividir y definir por rasgos esenciales absolutamente (simpliciter), y reunir (colligere) o demostrar por causas esenciales absolutamente anteriores y más conocidas, y en grado sumo por las causas más altas.

27. De este modo sería cognoscible la metafísica por sí. Sin embargo, nosotros no la conocemos así, ni así es transmitida por Aristóteles, puesto que, debido a la impotencia de nuestro intelecto, a partir de las cosas sensibles y menos conocidas por sí, alcanzamos el conocimiento de las realidades inmateriales, las cuales son por sí más conocidas y deberían ser tomadas, en la metafísica, como principios de conocer las otras.

28. [Adición posterior: §§ 28-31] ใ Se pregunta: ¿Cuál causa es el sujeto (subiectum) de esta ciencia?

Al parecer es la causa efectiva (effectiva), pues se encuentra en el mismo género de la causa aquello que se relaciona con la potencia y aquello que se relaciona con su hábito (por ejemplo: como el bien es fin de la voluntad y de la caridad), pues el hábito no modifica el concepto del objeto de la potencia, sino que le da el modo de operar. Ahora bien, el objeto inteligible parece ser la causa eficiente con respecto a la potencia intelectiva, puesto que esta es pasiva.

29. Sin embargo, al parecer <el sujeto> es el fin de la ciencia, ya que el objeto de la voluntad es su fin, y por lo tanto, también lo es de la ciencia. Prueba de esta implicación: aquello 
que es fin del fin, es un fin de aquello ordenado al fin. El acto de la voluntad es fin del acto del intelecto. - Además, se busca (intenditur) principalmente el conocimiento del sujeto (cognitio subiecti), de lo contrario no sería una ciencia, sino muchas. - Además, cada cosa se denomina de acuerdo a su fin, y la ciencia de acuerdo a su sujeto. Por lo tanto, etc.

30. Sin embargo, al parecer <el sujeto>, con respecto a la ciencia, tiene la razón de causa formal, puesto que el sujeto le da la especie, la unidad, el orden y la dignidad; ahora bien, estas se encuentran en la forma.

31. Sin embargo, se dice comunmente que <el sujeto $>$ tiene la razón de materia. - Pero hay una diferencia: pues el sujeto es conocido previamente (praecognoscitur) confusamente, y se busca <conocerlo> distintamente (distincte). La materia de ningún modo puede conocerse previamente en acto, como ocurre con la materia y el sujeto en las ciencias naturales. Asimismo, la ciencia tiene su unidad, distinción, orden y necesidad a partir del sujeto, no a partir de la materia. १ใ

32. Hay que subrayar a propósito de la causa material, que no hablamos de la materia de la ciencia como «aquello a partir de la cual» (ex qua), puesto que los accidentes no tienen tal materia (según el Filósofo en el libro VIII, capítulo 5), ${ }^{29}$ ni de la materia «en la cual» (esta es, en efecto, el alma, y esto debido a su potencia intelectiva), sino que hablamos de la materia como «aquello a propósito de lo cual» hay ciencia, y algunos ${ }^{30}$ llaman a esta materia el sujeto de la ciencia, o más propiamente objeto, así como aquello a propósito de lo cual hay virtud, es llamado propiamente objeto de la virtud, y no sujeto.

\section{BIBLIOGRAFÍA}

Agustín de Hipona, De diversis quaestionibus octoginta tribus, De octo dulcitii quaestionibus, ed. A. Mutzenbecher, «Corpus Christianorum, Series Latina XLIV A», Turnhout, Brepols, 1975.

Aristoteles Latinus, De Anima, ed. R.-A. Gauthier, en Tomás de Aquino, Sententia libri De

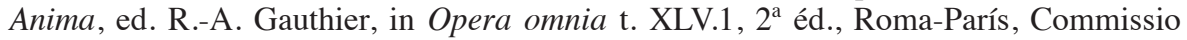
Leonina-J. Vrin, 1984.

- Ethica Nicomachea. Translatio Roberti Grosseteste Lincolniensis sive «Liber Ethicorum» A. Recensio Pura, ed. R.-A. Gauthier, «Aristoteles Latinus, XVI 1-3: Fasciculus Tertius», Leyde-Bruxelles, Brill-Desclée de Brouwer, 1972.

- Metaphysica, lib. I-X, XII-XIV. Translatio Anonyma sive «Media», ed. G. VuilleminDiem, «Aristoteles Latinus, XXV 2», Leyden, E.J. Brill, 1976.

- Physica. Translatio Vetus, eds. F. Bossier y J. Brams, Translatio Vaticana, ed. A. Mansion, 2 vols, 2 ed, «Aristoteles Latinus, VII 1-2», Leyde-New York, Brill, 1990.

Avicena, Liber de philosophia prima sive Scientia divina I-IV, ed. S. Van Riet ; introduction doctrinale G. Verbeke, «Avicenna Latinus», Louvain-Leyde, Peeters-Brill, 1977.

Enrique de Gante, Summae Quaestionum Ordinariarum (Reimpresión de la edición de 1520), II vols., ed. Eligius M. Buytaert, «Franciscan Institute Publications, Texts Series 1», St. Bonaventure (NY)-Louvain-Padenborn, The Franciscan Institute-E. Nauwelaerts-F. Shöningh, 1953.

29 Aristóteles, Metaphysica, AL XXV² p. 167; VIII (H) c. 5, 1045b 23-24.

30 Enrique de Gante, Summa, a. 19, q. 1 ad 2 (I, f. 115 H-I) y también, Tomás de Aquino, Summa theologicae, Ia-IIae, q. 55, a. 4, respondeo (edición Leonina, VI, 353). 
Juan Damasceno, De Fide Orthodoxa, Versions of Burgundio and Cerbanus, ed. E. M. Buytaert, «Franciscan Institute Publications, Texts Series 8», St. Bonaventure (NY)Louvain-Padenborn, The Franciscan Institute-E. Nauwelaerts-F. Shöningh, 1955.

Juan Duns Escoto, Opera philosophica III-IV: Quaestiones super libros metaphysicorum Aristotelis, ed. R. Andrews et al., St. Bonaventure (NY), The Franciscan Institute, 1997.

Rabano Mauro, Expositio in epistolem I ad Corinthios, ed. J.-P. Migne, «Patrologia Latina, 112», París, 1847.

Tomás de Aquino, In Duodecim Libros Metaphysicorum Aristotelis expositio, eds. M. R. Cathala y R. M. Spiazzi, Turín-Roma, Marietti, 1950. 